\title{
Undercarboxylated osteocalcin may be an attractive marker of teriparatide treatment in rheumatoid arthritis patients
}

\author{
S. Mokuda
}

Received: 30 September 2014 / Accepted: 28 October 2014 / Published online: 13 November 2014

(C) International Osteoporosis Foundation and National Osteoporosis Foundation 2014

Dear Editor,

Elderly rheumatoid arthritis (RA) patients suffer from osteoporosis resulting in fragility fractures [1]. Therapy with lowdose glucocorticoids, which are often used to treat RA, suppresses bone strength. However, evidence regarding the effects of treatment for osteoporosis in RA patients remains limited. Ebina and colleagues [2] conducted an 18-month prospective trial of daily teriparatide (TPTD) in patients with RA and postmenopausal osteoporosis. Their findings showed the advantage of TPTD in the RA patients, compared to that observed in the subjects with primary osteoporosis, and identified available predictors of TPTD treatment in the setting of RA. Their findings provide several significant messages for clinicians. In the current article, I would like to discuss this topic from the point of view of a marker of bone formation, undercarboxylated osteocalcin (ucOC).

It has previously been reported that the serum ucOC level is elevated by TPTD treatment in RA patients [3]. In addition, serum ucOC level and daily glucocorticoid dose are inversely correlated in RA patients treated with teriparatide [3]. The serum ucOC levels in RA patients receiving more than $10 \mathrm{mg}$ per day of prednisolone are often undetectable on an electrochemiluminescence immunoassay. We recently administered TPTD treatment in a small number of RA patients and followed the BMD and serum ucOC levels for 48 weeks ( $n=$ 34 , mean age 74.6 years). Based on our preliminary data, the percent change from baseline in the LS BMD at 48 weeks positively correlated with the absolute change from baseline in
ucOC at 24 weeks ( $\mathrm{rs}=0.389, p=0.025)$. These results are consistent with the observations noted by Ebina and colleagues [2]. Therefore, we agree with the suggestion that the serum ucOC level has the potential to be a useful predictor of TPTD treatment in RA patients.

Nevertheless, it is unknown whether the ucOC level is a suitable biomarker for TPTD treatment in all types of glucocorticoid-induced osteoporosis (GIO). As noted above, high-dose prednisolone treatment may make the serum ucOC level undetectable. Therefore, further research is needed to clarify the relationship between ucOC and TPTD treatment in RA and GIO patients.

\section{References}

1. Roux C (2011) Osteoporosis in inflammatory joint diseases. Osteoporos Int 22:421-433

2. Ebina K, Hashimoto J, Shi K, Kashii M, Hirao M, Yoshikawa $\mathrm{H}$ (2014) Comparison of the effect of 18-month daily teriparatide administration on patients with rheumatoid arthritis and postmenopausal osteoporosis patients. Osteoporos Int 25: 2755-2765. doi:10.1007/s00198-014-2819-x

3. Mokuda S, Sawada N, Matoba K, Yamada A, Onishi M, Okuda Y, Jouyama K, Murata Y, Takasugi K (2012) Serum undercarboxylated osteocalcin level increases with 48 weeks of teriparatide treatment in pre-treated elderly rheumatoid arthritis patients who use anti-resorptive drugs. J Endocrinol Invest 35: 796-799
S. Mokuda $(\bowtie)$

Department of Internal Medicine, Center for Rheumatic Diseases,

Dohgo Spa Hospital, 21-21 Otsu Dohgo-Himezuka, Matsuyama,

Ehime 790-0858, Japan

e-mail: sho-mokuda@hiroshima-u.ac.jp 